\title{
A new multi-step iteration for solving a fixed point problem of nonexpansive mappings
}

\section{Prasit Cholamjiak}

\section{"Correspondence:}

prasitch2008@yahoo.com

School of Science, University of

Phayao, Phayao, 56000, Thailand

\begin{abstract}
We introduce a new nonlinear mapping generated by a finite family of nonexpansive mappings. Weak and strong convergence theorems are also established in the setting of a Banach space.

MSC: $47 \mathrm{H} 09 ; 47 \mathrm{H} 10$

Keywords: nonexpansive mapping; weak convergence; Banach space; fixed point; strong convergence
\end{abstract}

\section{Introduction}

Let $C$ be a nonempty, closed and convex subset of a real Banach space $E$. Let $T: C \rightarrow C$ be a nonlinear mapping. The fixed point set of $T$ is denoted by $F(T)$, that is, $F(T)=\{x \in$ $C: x=T x\}$. Recall that a mapping $T$ is said to be nonexpansive if $\|T x-T y\| \leq\|x-y\|$ for all $x, y \in C$, and a mapping $f: C \rightarrow C$ is called a contraction if there exists a constant $\alpha \in(0,1)$ such that $\|f(x)-f(y)\| \leq \alpha\|x-y\|$ for all $x, y \in C$. We use $\Sigma_{C}$ to denote a class of contractions with constant $\alpha$.

Fixed point problems are now arising in a wide range of applications such as optimization, physics, engineering, economics and applied sciences. Many related problems can be cast as the problem of finding fixed points for nonlinear mappings. The interdisciplinary nature of fixed point problems is evident through a vast literature which includes a large body of mathematical and algorithmic developments.

In the literature, several types of iterations have been constructed and proposed in order to get convergence results for nonexpansive mappings in various settings. One classical iteration process is defined as follows: $x_{1} \in C$ and

$$
x_{n+1}=\left(1-\alpha_{n}\right) x_{n}+\alpha_{n} T x_{n}, \quad \forall n \geq 1,
$$

where $\left\{\alpha_{n}\right\} \subset(0,1)$. This method was introduced in 1953 by Mann [1] and is known as the Mann iteration process. However, we note that it has only weak convergence in general; for instance, see [2].

In 1974, Ishikawa [3] proposed the following two-step iteration: $x_{1} \in C$ and

$$
\begin{aligned}
& y_{n}=\left(1-\beta_{n}\right) x_{n}+\beta_{n} T x_{n}, \\
& x_{n+1}=\left(1-\alpha_{n}\right) x_{n}+\alpha_{n} T y_{n}, \quad \forall n \geq 1,
\end{aligned}
$$

○ 2013 Cholamjiak; licensee Springer. This is an Open Access article distributed under the terms of the Creative Commons Attribution License (http://creativecommons.org/licenses/by/2.0), which permits unrestricted use, distribution, and reproduction in any medium, provided the original work is properly cited. 
where $\left\{\alpha_{n}\right\}$ and $\left\{\beta_{n}\right\}$ are sequences in $(0,1)$. This method is often called the Ishikawa iteration process.

Very recently, Agarwal et al. [4] introduced a new iteration process as follows: $x_{1} \in C$ and

$$
\begin{aligned}
& y_{n}=\left(1-\beta_{n}\right) x_{n}+\beta_{n} T x_{n}, \\
& x_{n+1}=\left(1-\alpha_{n}\right) T x_{n}+\alpha_{n} T y_{n}, \quad \forall n \geq 1,
\end{aligned}
$$

where $\left\{\alpha_{n}\right\}$ and $\left\{\beta_{n}\right\}$ are sequences in $(0,1)$. This method is called the $S$-iteration process. The weak convergence was studied in [5] for nonexpansive mappings. It was also shown in [5] that the convergence rate of the $S$-iteration process is faster than the Picard and Mann iteration processes for contractive mappings.

Firstly, motivated by Agarwal et al. [4], we have the aim to introduce and study a new mapping defined by the following definition.

Definition 1.1 Let $C$ be a nonempty and convex subset of a real Banach space $E$. Let $T_{1}, T_{2}, \ldots, T_{N}$ be a finite family of nonexpansive mappings of $C$ into itself, and let $\lambda_{1}, \lambda_{2}, \ldots, \lambda_{N}$ be real numbers such that $0 \leq \lambda_{i} \leq 1$ for all $i=1,2, \ldots, N$. Define the mapping $B: C \rightarrow C$ as follows:

$$
\begin{aligned}
& U_{1}=\lambda_{1} T_{1}+\left(1-\lambda_{1}\right) I, \\
& U_{2}=\lambda_{2} T_{2} U_{1}+\left(1-\lambda_{2}\right) T_{1}, \\
& U_{3}=\lambda_{3} T_{3} U_{2}+\left(1-\lambda_{3}\right) T_{2}, \\
& \vdots \\
& U_{N-1}=\lambda_{N-1} T_{N-1} U_{N-2}+\left(1-\lambda_{N-1}\right) T_{N-2}, \\
& B=U_{N}=\lambda_{N} T_{N} U_{N-1}+\left(1-\lambda_{N}\right) T_{N-1} .
\end{aligned}
$$

Such a mapping $B$ is called the $B$-mapping generated by $T_{1}, T_{2}, \ldots, T_{N}$ and $\lambda_{1}, \lambda_{2}, \ldots, \lambda_{N}$. See [6-9] for the corresponding concept.

Secondly, using the definition above, we study weak convergence of the following Manntype iteration process in a uniformly convex Banach space with a Fréchet differentiable norm or that satisfies Opial's condition: $x_{1} \in C$ and

$$
x_{n+1}=\left(1-\alpha_{n}\right) x_{n}+\alpha_{n} B_{n} x_{n}, \quad \forall n \geq 1,
$$

where $B_{n}$ is a $B$-mapping generated by $T_{1}, T_{2}, \ldots, T_{N}$ and $\lambda_{n, 1}, \lambda_{n, 2}, \ldots, \lambda_{n, N}$ (see Section 3 ).

Finally, we discuss strong convergence of the iteration scheme involving the modified viscosity approximation method [10] defined as follows: $x_{1} \in C$ and

$$
x_{n+1}=\alpha_{n} f\left(x_{n}\right)+\beta_{n} x_{n}+\gamma_{n} B_{n} x_{n}, \quad \forall n \geq 1,
$$

where $\left\{\alpha_{n}\right\},\left\{\beta_{n}\right\}$ and $\left\{\gamma_{n}\right\}$ are sequences in $(0,1)$, and $f \in \Sigma_{C}$.

More references on earlier works promoting the theory of fixed points and common fixed points for nonexpansive mappings can be found in [11-21]. 
Throughout this paper, we use the notation:

- $\rightarrow$ for weak convergence and $\rightarrow$ for strong convergence.

- $\omega_{\omega}\left(x_{n}\right)=\left\{x: x_{n_{i}} \rightarrow x\right\}$ denotes the weak $\omega$-limit set of $\left\{x_{n}\right\}$.

\section{Preliminaries and lemmas}

In this section, we begin by recalling some basic facts and lemmas which will be used in the sequel.

Let $E$ be a real Banach space and let $U=\{x \in E:\|x\|=1\}$ be the unit sphere of $E$. A Banach space $E$ is said to be strictly convex if for any $x, y \in U$,

$$
x \neq y \text { implies }\left\|\frac{x+y}{2}\right\|<1 .
$$

It is also said to be uniformly convex if for each $\varepsilon \in(0,2]$, there exists $\delta>0$ such that for any $x, y \in U$,

$$
\|x-y\| \geq \varepsilon \quad \text { implies } \quad\left\|\frac{x+y}{2}\right\|<1-\delta .
$$

It is known that a uniformly convex Banach space is reflexive and strictly convex. Define a function $\delta:[0,2] \rightarrow[0,1]$ called the modulus of convexity of $E$ as follows:

$$
\delta(\varepsilon)=\inf \left\{1-\left\|\frac{x+y}{2}\right\|: x, y \in E,\|x\|=\|y\|=1,\|x-y\| \geq \varepsilon\right\} .
$$

Then $E$ is uniformly convex if and only if $\delta(\varepsilon)>0$ for all $\varepsilon \in(0,2]$. A Banach space $E$ is said to be smooth if the limit

$$
\lim _{t \rightarrow 0} \frac{\|x+t y\|-\|x\|}{t}
$$

exists for all $x, y \in U$. The norm is said to be uniformly Gâteaux differentiable if for $y \in U$, the limit is attained uniformly for $x \in U$. It is said to be Fréchet differentiable if for $x \in U$, the limit is attained uniformly for $y \in U$. It is said to be uniformly smooth or uniformly Fréchet differentiable if the limit (2.1) is attained uniformly for $x, y \in U$. The normalized duality mapping $J: E \rightarrow 2^{E^{*}}$ is defined by

$$
J(x)=\left\{x^{*} \in E^{*}:\left\langle x, x^{*}\right\rangle=\|x\|^{2}=\left\|x^{*}\right\|^{2}\right\}
$$

for all $x \in E$. It is known that $E$ is smooth if and only if the duality mapping $J$ is single valued, and that if $E$ has a uniformly Gâteaux differentiable norm, $J$ is uniformly norm-toweak* continuous on each bounded subset of $E$. A Banach space $E$ is said to satisfy Opial's condition [22]. If $x \in E$ and $x_{n} \rightarrow x$, then

$$
\limsup _{n \rightarrow \infty}\left\|x_{n}-x\right\|<\limsup _{n \rightarrow \infty}\left\|x_{n}-y\right\|, \quad \forall y \in E, x \neq y .
$$

Let $T: C \rightarrow C$. Then $I-T$ is demiclosed at 0 if for all sequence $\left\{x_{n}\right\}$ in $C, x_{n} \rightarrow q$ and $\left\|x_{n}-T x_{n}\right\| \rightarrow 0$ imply $q=T q$. It is known that if $E$ is uniformly convex, $C$ is nonempty closed and convex, and $T$ is nonexpansive, then $I-T$ is demiclosed at 0 [23]. For more details, we refer the reader to $[5,24]$. 
Lemma 2.1 [5] Let E be a smooth Banach space. Then the following hold:

(i) $\|x+y\|^{2} \geq\|x\|^{2}+2\langle y, J(x)\rangle$ for all $x, y \in E$;

(ii) $\|x+y\|^{2} \leq\|x\|^{2}+2\langle y, J(x+y)\rangle$ for all $x, y \in E$.

Lemma 2.2 [24] In a strictly convex Banach space E, if

$$
\|x\|=\|y\|=\|\lambda x+(1-\lambda) y\|
$$

for all $x, y \in E$ and $\lambda \in(0,1)$, then $x=y$.

Lemma 2.3 [25] Let $\left\{x_{n}\right\}$ and $\left\{z_{n}\right\}$ be two sequences in a Banach space E such that

$$
x_{n+1}=\beta_{n} x_{n}+\left(1-\beta_{n}\right) z_{n}, \quad n \geq 1,
$$

where $\left\{\beta_{n}\right\}$ satisfies the conditions $0<\liminf _{n \rightarrow \infty} \beta_{n} \leq \limsup _{n \rightarrow \infty} \beta_{n}<1$.

$$
\text { If } \lim \sup _{n \rightarrow \infty}\left(\left\|z_{n+1}-z_{n}\right\|-\left\|x_{n+1}-x_{n}\right\|\right) \leq 0 \text {, then }\left\|z_{n}-x_{n}\right\| \rightarrow 0 \text { as } n \rightarrow \infty \text {. }
$$

Lemma 2.4 [26] Let E be a uniformly convex Banach space with a Fréchet differentiable norm. Let $C$ be a closed and convex subset of $E$, and let $\left\{S_{n}\right\}_{n=1}^{\infty}$ be a family of $L_{n}$-Lipschitzian self-mappings on $C$ such that $\sum_{n=1}^{\infty}\left(L_{n}-1\right)<\infty$ and $F=\bigcap_{n=1}^{\infty} F\left(S_{n}\right) \neq \emptyset$. For arbitrary $x_{1} \in$ $C$, define $x_{n+1}=S_{n} x_{n}$ for all $n \geq 1$. Then, for every $p, q \in F, \lim _{n \rightarrow \infty}\left\langle x_{n}, J(p-q)\right\rangle$ exists, in particular, for all $u, v \in \omega_{\omega}\left(x_{n}\right)$ and $p, q \in F,\langle u-v, J(p-q)\rangle=0$.

Lemma 2.5 [15] Let $E$ be a reflexive and strictly convex Banach space with a uniformly Gâteaux differentiable norm, let $C$ be a nonempty closed convex subset of $E$, let $A: C \rightarrow C$ be a continuous strongly pseudocontractive mapping with constant $k \in[0,1)$, and let $T$ : $C \rightarrow E$ be a continuous pseudocontractive mapping satisfying the weakly inward condition. If $T$ has a fixed point in $C$, then the path $\left\{x_{t}\right\}$ defined by

$$
x_{t}=t A x_{t}+(1-t) T x_{t}
$$

converges strongly to a fixed point $q$ of $T$ as $t \rightarrow 0$, which is a unique solution of the variational inequality

$$
\langle(I-A) q, J(q-p)\rangle \leq 0, \quad \forall p \in F(T) .
$$

Remark 2.6 Lemma 2.5 holds if $T: C \rightarrow C$ is a nonexpansive mapping and $A=f$ is a contraction.

The following lemma gives us a nice property of real sequences.

Lemma 2.7 [18] Assume that $\left\{a_{n}\right\}$ is a sequence of nonnegative real numbers such that

$$
a_{n+1} \leq\left(1-c_{n}\right) a_{n}+b_{n}, \quad \forall n \geq 1,
$$

where $\left\{c_{n}\right\}$ is a sequence in $(0,1)$ and $\left\{b_{n}\right\}$ is a sequence such that

(a) $\sum_{n=1}^{\infty} c_{n}=\infty$;

(b) $\lim \sup _{n \rightarrow \infty} \frac{b_{n}}{c_{n}} \leq 0$ or $\sum_{n=1}^{\infty}\left|b_{n}\right|<\infty$.

Then $\lim _{n \rightarrow \infty} a_{n}=0$. 


\section{Weak convergence theorem}

In this section, we give some properties concerning the $B$-mapping and then prove a weak convergence theorem for nonexpansive mappings.

Lemma 3.1 Let $C$ be a nonempty, closed and convex subset of a strictly convex Banach space E. Let $\left\{T_{i}\right\}_{i=1}^{N}$ be a finite family of nonexpansive mappings of $C$ into itself such that $\bigcap_{i=1}^{N} F\left(T_{i}\right) \neq \emptyset$, and let $\lambda_{1}, \lambda_{2}, \ldots, \lambda_{N}$ be real numbers such that $0<\lambda_{i}<1$ for all $i=1,2, \ldots, N-1$ and $0<\lambda_{N} \leq 1$. Let $B$ be the B-mapping generated by $T_{1}, T_{2}, \ldots, T_{N}$ and $\lambda_{1}, \lambda_{2}, \ldots, \lambda_{N}$. Then the following hold:

(i) $F(B)=\bigcap_{i=1}^{N} F\left(T_{i}\right)$;

(ii) $B$ is nonexpansive.

Proof (i) Since $\bigcap_{i=1}^{N} F\left(T_{i}\right) \subset F(B)$ is trivial, it suffices to show that $F(B) \subset \bigcap_{i=1}^{N} F\left(T_{i}\right)$. To this end, let $p \in F(B)$ and $x^{*} \in \bigcap_{i=1}^{N} F\left(T_{i}\right)$. Then we have

$$
\begin{aligned}
\left\|p-x^{*}\right\|= & \left\|B p-x^{*}\right\|=\left\|\lambda_{N}\left(T_{N} U_{N-1} p-x^{*}\right)+\left(1-\lambda_{N}\right)\left(T_{N-1} p-x^{*}\right)\right\| \\
\leq & \lambda_{N}\left\|U_{N-1} p-x^{*}\right\|+\left(1-\lambda_{N}\right)\left\|p-x^{*}\right\| \\
= & \lambda_{N}\left\|\lambda_{N-1}\left(T_{N-1} U_{N-2} p-x^{*}\right)+\left(1-\lambda_{N-1}\right)\left(T_{N-2} p-x^{*}\right)\right\|+\left(1-\lambda_{N}\right)\left\|p-x^{*}\right\| \\
\leq & \lambda_{N} \lambda_{N-1}\left\|U_{N-2} p-x^{*}\right\|+\left(1-\lambda_{N} \lambda_{N-1}\right)\left\|p-x^{*}\right\| \\
= & \lambda_{N} \lambda_{N-1}\left\|\lambda_{N-2}\left(T_{N-2} U_{N-3} p-x^{*}\right)+\left(1-\lambda_{N-2}\right)\left(T_{N-3} p-x^{*}\right)\right\| \\
& +\left(1-\lambda_{N} \lambda_{N-1}\right)\left\|p-x^{*}\right\| \\
\leq & \lambda_{N} \lambda_{N-1} \lambda_{N-2}\left\|U_{N-3} p-x^{*}\right\|+\left(1-\lambda_{N} \lambda_{N-1} \lambda_{N-2}\right)\left\|p-x^{*}\right\| \\
\vdots & \\
= & \lambda_{N} \lambda_{N-1} \cdots \lambda_{3}\left\|\lambda_{2}\left(T_{2} U_{1} p-x^{*}\right)+\left(1-\lambda_{2}\right)\left(T_{1} p-x^{*}\right)\right\| \\
& +\left(1-\lambda_{N} \lambda_{N-1} \cdots \lambda_{3}\right)\left\|p-x^{*}\right\| \\
\leq & \lambda_{N} \lambda_{N-1} \cdots \lambda_{2}\left\|T_{2} U_{1} p-x^{*}\right\|+\left(1-\lambda_{N} \lambda_{N-1} \cdots \lambda_{2}\right)\left\|p-x^{*}\right\| \\
\leq & \lambda_{N} \lambda_{N-1} \cdots \lambda_{2}\left\|U_{1} p-x^{*}\right\|+\left(1-\lambda_{N} \lambda_{N-1} \cdots \lambda_{2}\right)\left\|p-x^{*}\right\| \\
= & \lambda_{N} \lambda_{N-1} \cdots \lambda_{2}\left\|\lambda_{1}\left(T_{1} p-x^{*}\right)+\left(1-\lambda_{1}\right)\left(p-x^{*}\right)\right\| \\
& +\left(1-\lambda_{N} \lambda_{N-1} \cdots \lambda_{2}\right)\left\|p-x^{*}\right\| \\
\leq & \lambda_{N} \lambda_{N-1} \cdots \lambda_{2} \lambda_{1}\left\|T_{1} p-x^{*}\right\|+\left(1-\lambda_{N} \lambda_{N-1} \cdots \lambda_{2} \lambda_{1}\right)\left\|p-x^{*}\right\| \\
\leq & \lambda_{N} \lambda_{N-1} \cdots \lambda_{2} \lambda_{1}\left\|p-x^{*}\right\|+\left(1-\lambda_{N} \lambda_{N-1} \cdots \lambda_{2} \lambda_{1}\right)\left\|p-x^{*}\right\| \\
= & \left\|p-x^{*}\right\| . \\
& \\
& \\
&
\end{aligned}
$$

This shows that

$$
\left\|p-x^{*}\right\|=\lambda_{N} \lambda_{N-1} \cdots \lambda_{2}\left\|\lambda_{1}\left(T_{1} p-x^{*}\right)+\left(1-\lambda_{1}\right)\left(p-x^{*}\right)\right\|+\left(1-\lambda_{N} \lambda_{N-1} \cdots \lambda_{2}\right)\left\|p-x^{*}\right\|,
$$

which turns out to be

$$
\left\|p-x^{*}\right\|=\left\|\lambda_{1}\left(T_{1} p-x^{*}\right)+\left(1-\lambda_{1}\right)\left(p-x^{*}\right)\right\| .
$$


Again by (3.1), we see that $\left\|p-x^{*}\right\|=\left\|T_{1} p-x^{*}\right\|$ and thus

$$
\left\|p-x^{*}\right\|=\left\|T_{1} p-x^{*}\right\|=\left\|\lambda_{1}\left(T_{1} p-x^{*}\right)+\left(1-\lambda_{1}\right)\left(p-x^{*}\right)\right\| .
$$

Using Lemma 2.2, we get that $T_{1} p=p$ and hence $U_{1} p=p$. Again by (3.1), we have

$$
\begin{aligned}
\left\|p-x^{*}\right\|= & \lambda_{N} \lambda_{N-1} \cdots \lambda_{3}\left\|\lambda_{2}\left(T_{2} U_{1} p-x^{*}\right)+\left(1-\lambda_{2}\right)\left(T_{1} p-x^{*}\right)\right\| \\
& +\left(1-\lambda_{N} \lambda_{N-1} \cdots \lambda_{3}\right)\left\|p-x^{*}\right\|
\end{aligned}
$$

which implies that

$$
\left\|p-x^{*}\right\|=\left\|\lambda_{2}\left(T_{2} U_{1} p-x^{*}\right)+\left(1-\lambda_{2}\right)\left(T_{1} p-x^{*}\right)\right\| .
$$

From (3.1) we see that $\left\|U_{1} p-x^{*}\right\|=\left\|T_{2} U_{1} p-x^{*}\right\|$. Since $U_{1} p=p$ and $T_{1} p=p$,

$$
\left\|p-x^{*}\right\|=\left\|T_{2} p-x^{*}\right\|=\left\|\lambda_{2}\left(T_{2} p-x^{*}\right)+\left(1-\lambda_{2}\right)\left(p-x^{*}\right)\right\| .
$$

Using Lemma 2.2, we get that $T_{2} p=p$ and hence $U_{2} p=p$.

By continuing this process, we can show that $T_{i} p=p$ and $U_{i} p=p$ for all $i=1,2, \ldots, N-1$. Finally, we obtain

$$
\begin{aligned}
\left\|p-T_{N} p\right\| & \leq\|p-B p\|+\left\|B p-T_{N} p\right\| \\
& =\|p-B p\|+\left(1-\lambda_{N}\right)\left\|p-T_{N} p\right\|,
\end{aligned}
$$

which yields that $p=T_{N} p$ since $p \in F(B)$. Hence $p=T_{1} p=T_{2} p=\cdots=T_{N} p$ and thus $p \in$ $\bigcap_{i=1}^{N} F\left(T_{i}\right)$.

(ii) The proof follows directly from (i).

Lemma 3.2 Let $C$ be a nonempty closed convex subset of a real Banach space E. Let $\left\{T_{i}\right\}_{i=1}^{N}$ be a finite family of nonexpansive mappings of $C$ into itself such that $\bigcap_{i=1}^{N} F\left(T_{i}\right) \neq \emptyset$, and let $B$ be the B-mapping generated by $T_{1}, T_{2}, \ldots, T_{N}$ and $\lambda_{1}, \lambda_{2}, \ldots, \lambda_{N}$. Let $\left\{\lambda_{n, i}\right\}_{i=1}^{N}$ be a real sequence in $(0,1)$. For every $n \in \mathbb{N}$, let $B_{n}$ be the B-mapping generated by $T_{1}, T_{2}, \ldots, T_{N}$ and $\lambda_{n, 1}, \lambda_{n, 2}, \ldots, \lambda_{n, N}$ as follows:

$$
\begin{aligned}
& U_{n, 1}=\lambda_{n, 1} T_{1}+\left(1-\lambda_{n, 1}\right) I, \\
& U_{n, 2}=\lambda_{n, 2} T_{2} U_{1}+\left(1-\lambda_{n, 2}\right) T_{1}, \\
& U_{n, 3}=\lambda_{n, 3} T_{3} U_{2}+\left(1-\lambda_{n, 3}\right) T_{2}, \\
& \vdots \\
& U_{n, N-1}=\lambda_{n, N-1} T_{N-1} U_{N-2}+\left(1-\lambda_{n, N-1}\right) T_{N-2}, \\
& B_{n}=U_{n, N}=\lambda_{n, N} T_{N} U_{N-1}+\left(1-\lambda_{n, N}\right) T_{N-1} .
\end{aligned}
$$

If $\lambda_{n, i} \rightarrow \lambda_{i} \in(0,1)$ for all $i=1,2, \ldots, N$, then $\lim _{n \rightarrow \infty} B_{n} x=B x$ for all $x \in C$. 
Proof Let $x \in C$ and $U_{k}$ and $U_{n, k}$ be generated by $T_{1}, T_{2}, \ldots, T_{k}$ and $\lambda_{1}, \lambda_{2}, \ldots, \lambda_{k}$, and $T_{1}, T_{2}, \ldots, T_{k}$ and $\lambda_{n, 1}, \lambda_{n, 2}, \ldots, \lambda_{n, k}$, respectively. Then

$$
\left\|U_{n, 1} x-U_{1} x\right\|=\left\|\left(\lambda_{n, 1}-\lambda_{1}\right)\left(T_{1} x-x\right)\right\| \leq\left|\lambda_{n, 1}-\lambda_{1}\right|\left\|T_{1} x-x\right\| .
$$

Let $k \in\{2,3, \ldots, N\}$ and $M=\max \left\{\left\|T_{k} U_{k-1} x\right\|+\left\|T_{k-1} x\right\|: k=2,3, \ldots, N\right\}$. Then

$$
\begin{aligned}
\left\|U_{n, k} x-U_{k} x\right\|= & \left\|\lambda_{n, k} T_{k} U_{n, k-1} x+\left(1-\lambda_{n, k}\right) T_{k-1} x-\lambda_{k} T_{k} U_{k-1}-\left(1-\lambda_{k}\right) T_{k-1} x\right\| \\
= & \left\|\lambda_{n, k} T_{k} U_{n, k-1} x-\lambda_{n, k} T_{k-1} x-\lambda_{k} T_{k} U_{k-1}+\lambda_{k} T_{k-1} x\right\| \\
\leq & \lambda_{n, k}\left\|T_{k} U_{n, k-1} x-T_{k} U_{k-1} x\right\|+\left|\lambda_{n, k}-\lambda_{k}\right|\left\|T_{k} U_{k-1} x\right\| \\
& +\left|\lambda_{n, k}-\lambda_{k}\right|\left\|T_{k-1} x\right\| \\
\leq & \left\|U_{n, k-1} x-U_{k-1} x\right\|+\left|\lambda_{n, k}-\lambda_{k}\right| M .
\end{aligned}
$$

It follows that

$$
\begin{aligned}
\left\|B_{n} x-B x\right\|= & \left\|U_{n, N} x-U_{N} x\right\| \\
\leq & \left\|U_{n, N-1} x-U_{N-1} x\right\|+\left|\lambda_{n, N}-\lambda_{N}\right| M \\
\leq & \left\|U_{n, N-2} x-U_{N-2} x\right\|+\left|\lambda_{n, N-1}-\lambda_{N-1}\right| M+\left|\lambda_{n, N}-\lambda_{N}\right| M \\
& \vdots \\
\leq & \left\|U_{n, 1} x-U_{1} x\right\|+\left|\lambda_{n, 2}-\lambda_{2}\right| M+\cdots+\left|\lambda_{n, N-1}-\lambda_{N-1}\right| M+\left|\lambda_{n, N}-\lambda_{N}\right| M \\
\leq & \left|\lambda_{n, 1}-\lambda_{1}\right|\left\|T_{1} x-x\right\|+\left|\lambda_{n, 2}-\lambda_{2}\right| M+\cdots+\left|\lambda_{n, N-1}-\lambda_{N-1}\right| M \\
& +\left|\lambda_{n, N}-\lambda_{N}\right| M .
\end{aligned}
$$

Since $\lambda_{n, i} \rightarrow \lambda_{i}$ as $n \rightarrow \infty(i=1,2, \ldots, N)$, we thus complete the proof.

Remark 3.3 It is easily seen that for all $n \in \mathbb{N}, B_{n}$ is nonexpansive.

Lemma 3.4 Let $C$ be a nonempty closed convex subset of a real Banach space E. Let $\left\{T_{i}\right\}_{i=1}^{N}$ be a finite family of nonexpansive mappings of $C$ into itself such that $\bigcap_{i=1}^{N} F\left(T_{i}\right) \neq \emptyset$. Let $\left\{\lambda_{n, i}\right\}_{i=1}^{N}$ be a real sequence in $(0,1)$. For every $n \in \mathbb{N}$, let $B_{n}$ be the B-mapping generated by $T_{1}, T_{2}, \ldots, T_{N}$ and $\lambda_{n, 1}, \lambda_{n, 2}, \ldots, \lambda_{n, N}$.

$$
\text { If } \lim _{n \rightarrow \infty}\left|\lambda_{n+1, i}-\lambda_{n, i}\right|=0 \text { for all } i=1,2, \ldots, N \text {, then }
$$

$$
\lim _{n \rightarrow \infty}\left\|B_{n+1} z_{n}-B_{n} z_{n}\right\|=0
$$

for each bounded sequence $\left\{z_{n}\right\}$ in $C$.

Proof Let $\left\{z_{n}\right\}$ be a bounded sequence in $C$. For $j \in\{0,1, \ldots, N-2\}$ and for some $M>0$, we have

$$
\begin{aligned}
& \left\|U_{n+1, N-j} z_{n}-U_{n, N-j} z_{n}\right\| \\
& \quad=\| \lambda_{n+1, N-j} T_{N-j} U_{n+1, N-j-1} z_{n}+\left(1-\lambda_{n+1, N-j}\right) T_{N-j-1} z_{n}
\end{aligned}
$$




$$
\begin{aligned}
& -\lambda_{n, N-j} T_{N-j} U_{n, N-j-1} z_{n}-\left(1-\lambda_{n, N-j}\right) T_{N-j-1} z_{n} \| \\
\leq & \lambda_{n+1, N-j}\left\|T_{N-j} U_{n+1, N-j-1} z_{n}-T_{N-j} U_{n, N-j-1} z_{n}\right\| \\
& +\left|\lambda_{n+1, N-j}-\lambda_{n, N-j}\right|\left\|T_{N-j} U_{n, N-j-1} z_{n}\right\| \\
& +\left|\lambda_{n+1, N-j}-\lambda_{n, N-j}\right|\left\|T_{N-j-1} z_{n}\right\| \\
\leq & \left\|U_{n+1, N-j-1} z_{n}-U_{n, N-j-1} z_{n}\right\|+\left|\lambda_{n+1, N-j}-\lambda_{n, N-j}\right| M .
\end{aligned}
$$

Using the relation above, we can show that

$$
\begin{aligned}
\left\|B_{n+1} z_{n}-B_{n} z_{n}\right\| & =\left\|U_{n+1, N} z_{n}-U_{n, N} z_{n}\right\| \\
& \leq M \sum_{j=2}^{N}\left|\lambda_{n+1, j}-\lambda_{n, j}\right|+\left|\lambda_{n+1,1}-\lambda_{n, 1}\right|\left(\left\|z_{n}\right\|+\left\|T_{1} z_{n}\right\|\right) .
\end{aligned}
$$

Since $\lim _{n \rightarrow \infty}\left|\lambda_{n+1, i}-\lambda_{n, i}\right|=0$ for all $i=1,2, \ldots, N$, we obtain the desired result.

Using the concept of $B$-mapping, we study weak convergence of the sequence generated by Mann-type iteration process (1.2).

Theorem 3.5 Let E be a uniformly convex Banach space having a Fréchet differentiable norm or that satisfies Opial's condition. Let $C$ be a nonempty, closed and convex subset of E. Let $\left\{T_{i}\right\}_{i=1}^{N}$ be a finite family of nonexpansive mappings of $C$ into itself such that $\bigcap_{i=1}^{N} F\left(T_{i}\right) \neq \emptyset$. Let $\left\{\lambda_{n, i}\right\}_{i=1}^{N}$ be a real sequence in $(0,1)$ such that $\lambda_{n, i} \rightarrow \lambda_{i}(i=1,2, \ldots, N)$. For every $n \in \mathbb{N}$, let $B_{n}$ be the B-mapping generated by $T_{1}, T_{2}, \ldots, T_{N}$ and $\lambda_{n, 1}, \lambda_{n, 2}, \ldots, \lambda_{n, N}$. Let $\left\{\alpha_{n}\right\}$ be a sequence in $(0,1)$ satisfying $\liminf _{n \rightarrow \infty} \alpha_{n}\left(1-\alpha_{n}\right)>0$. Let $\left\{x_{n}\right\}$ be generated by $x_{1} \in C$ and

$$
x_{n+1}=\left(1-\alpha_{n}\right) x_{n}+\alpha_{n} B_{n} x_{n}, \quad \forall n \geq 1 .
$$

Then $\left\{x_{n}\right\}$ converges weakly to $x^{*} \in \bigcap_{i=1}^{N} F\left(T_{i}\right)$.

Proof Let $p \in \bigcap_{i=1}^{N} F\left(T_{i}\right)$. Then $p=B_{n} p$ for all $n \geq 1$ and hence

$$
\left\|x_{n+1}-p\right\| \leq\left(1-\alpha_{n}\right)\left\|x_{n}-p\right\|+\alpha_{n}\left\|B_{n} x_{n}-p\right\| \leq\left\|x_{n}-p\right\|
$$

It follows that $\left\{\left\|x_{n}-p\right\|\right\}$ is nonincreasing; consequently, $\lim _{n \rightarrow \infty}\left\|x_{n}-p\right\|$ exists. Assume $\left\|x_{n}-p\right\|>0$. Since $E$ is uniformly convex, it follows (see, for example, [27]) that

$$
\left\|x_{n+1}-p\right\| \leq\left\|x_{n}-p\right\|\left\{1-2 \min \left\{\alpha_{n}, 1-\alpha_{n}\right\} \delta_{E}\left(\frac{\left\|x_{n}-B_{n} x_{n}\right\|}{\left\|x_{n}-p\right\|}\right)\right\}
$$

which implies that

$$
\begin{aligned}
\alpha_{n}\left(1-\alpha_{n}\right)\left\|x_{n}-p\right\| \delta_{E}\left(\frac{\left\|x_{n}-B_{n} x_{n}\right\|}{\left\|x_{n}-p\right\|}\right) & \leq \min \left\{\alpha_{n}, 1-\alpha_{n}\right\}\left\|x_{n}-p\right\| \delta_{E}\left(\frac{\left\|x_{n}-B_{n} x_{n}\right\|}{\left\|x_{n}-p\right\|}\right) \\
& \leq \frac{1}{2}\left\{\left\|x_{n}-p\right\|-\left\|x_{n+1}-p\right\|\right\} .
\end{aligned}
$$


Since $\lim _{n \rightarrow \infty}\left\|x_{n}-p\right\|$ exists and $\liminf _{n \rightarrow \infty} \alpha_{n}\left(1-\alpha_{n}\right)>0$, by the continuity of $\delta_{E}$, we have $\lim _{n \rightarrow \infty}\left\|x_{n}-B_{n} x_{n}\right\|=0$. Since $\lambda_{n, i} \rightarrow \lambda_{i}(i=1,2, \ldots, N)$, let the mapping $B: C \rightarrow C$ be generated by $T_{1}, T_{2}, \ldots, T_{N}$ and $\lambda_{1}, \lambda_{2}, \ldots, \lambda_{N}$. Then, by Lemma 3.2, we have $\lim _{n \rightarrow \infty} \| B_{n} x-$ $B x \|=0$ for all $x \in C$. So we have

$$
\begin{aligned}
\left\|x_{n}-B x_{n}\right\| & \leq\left\|x_{n}-B_{n} x_{n}\right\|+\left\|B_{n} x_{n}-B x_{n}\right\| \\
& \leq\left\|x_{n}-B_{n} x_{n}\right\|+\sup _{z \in\left\{x_{n}\right\}}\left\|B_{n} z-B z\right\| \\
& \rightarrow 0 .
\end{aligned}
$$

Since $B$ is nonexpansive and $E$ is uniformly convex, by the demiclosedness principle, $\omega_{\omega}\left(x_{n}\right) \subset F(B)$. Moreover, $F(B)=\bigcap_{i=1}^{N} F\left(T_{i}\right)$ by Lemma 3.1(i).

We next show that $\omega_{\omega}\left(x_{n}\right)$ is a singleton. Indeed, suppose that $x^{*}, y^{*} \in \omega_{\omega}\left(x_{n}\right) \subset$ $\bigcap_{i=1}^{N} F\left(T_{i}\right)$. Define $S_{n}: C \rightarrow C$ by

$$
S_{n} x=\left(1-\alpha_{n}\right) x+\alpha_{n} B_{n} x, \quad x \in C .
$$

Then $S_{n}$ is nonexpansive and $x^{*}, y^{*} \in \bigcap_{n=1}^{\infty} F\left(S_{n}\right)$. Using Lemma 2.4, we have $\lim _{n \rightarrow \infty}\left\langle x_{n}\right.$, $\left.J\left(x^{*}-y^{*}\right)\right\rangle$ exists. Suppose that $\left\{x_{n_{k}}\right\}$ and $\left\{x_{m_{k}}\right\}$ are subsequences of $\left\{x_{n}\right\}$ such that $x_{n_{k}} \rightarrow x^{*}$ and $x_{m_{k}} \rightarrow y^{*}$. Then

$$
\left\|x^{*}-y^{*}\right\|^{2}=\left\langle x^{*}-y^{*}, J\left(x^{*}-y^{*}\right)\right\rangle=\lim _{k \rightarrow \infty}\left\langle x_{n_{k}}-x_{m_{k}}, J\left(x^{*}-y^{*}\right)\right\rangle=0 .
$$

This shows that $x^{*}=y^{*}$.

Assume that $E$ satisfies Opial's condition. Let $x^{*}, y^{*} \in \omega_{\omega}\left(x_{n}\right)$ and $\left\{x_{n_{k}}\right\}$ and $\left\{x_{m_{k}}\right\}$ be subsequences of $\left\{x_{n}\right\}$ such that $x_{n_{k}} \rightarrow x^{*}$ and $x_{m_{k}} \rightarrow y^{*}$. If $x^{*} \neq y^{*}$, then

$$
\begin{aligned}
\lim _{n \rightarrow \infty}\left\|x_{n}-x^{*}\right\| & =\lim _{k \rightarrow \infty}\left\|x_{n_{k}}-x^{*}\right\|<\lim _{k \rightarrow \infty}\left\|x_{n_{k}}-y^{*}\right\|=\lim _{k \rightarrow \infty}\left\|x_{m_{k}}-y^{*}\right\| \\
& <\lim _{k \rightarrow \infty}\left\|x_{m_{k}}-x^{*}\right\|=\lim _{n \rightarrow \infty}\left\|x_{n}-x^{*}\right\|,
\end{aligned}
$$

which is a contradiction. It follows that $x^{*}=y^{*}$. Therefore $x_{n} \rightarrow x^{*} \in \bigcap_{i=1}^{N} F\left(T_{i}\right)$ as $n \rightarrow \infty$. This completes the proof.

\section{Strong convergence theorem}

In this section, we prove a strong convergence theorem for a finite family of nonexpansive mappings in Banach spaces.

Theorem 4.1 Let $E$ be a strictly convex and reflexive Banach space having a uniformly Gâteaux differentiable norm. Let $C$ be a nonempty, closed and convex subset of E. Let $\left\{T_{i}\right\}_{i=1}^{N}$ be a finite family of nonexpansive mappings of $C$ into itself such that $\bigcap_{i=1}^{N} F\left(T_{i}\right) \neq \emptyset$. Let $\left\{\lambda_{n, i}\right\}_{i=1}^{N}$ be a real sequence in $(0,1)$ such that $\lambda_{n, i} \rightarrow \lambda_{i}(i=1,2, \ldots, N)$. For every $n \in \mathbb{N}$, let $B_{n}$ be the B-mapping generated by $T_{1}, T_{2}, \ldots, T_{N}$ and $\lambda_{n, 1}, \lambda_{n, 2}, \ldots, \lambda_{n, N}$. Let $\left\{\alpha_{n}\right\},\left\{\beta_{n}\right\}$ and $\left\{\gamma_{n}\right\}$ be sequences in $(0,1)$ which satisfy the conditions:

(C1) $\alpha_{n}+\beta_{n}+\gamma_{n}=1$

(C2) $\lim _{n \rightarrow \infty} \alpha_{n}=0$ and $\sum_{n=1}^{\infty} \alpha_{n}=\infty$; 
(C3) $0<\liminf _{n \rightarrow \infty} \beta_{n} \leq \lim \sup _{n \rightarrow \infty} \beta_{n}<1$.

Let $f \in \Sigma_{C}$ and define the sequence $\left\{x_{n}\right\}$ by $x_{1} \in C$ and

$$
x_{n+1}=\alpha_{n} f\left(x_{n}\right)+\beta_{n} x_{n}+\gamma_{n} B_{n} x_{n}, \quad \forall n \geq 1 .
$$

Then $\left\{x_{n}\right\}$ converges strongly to $q \in \bigcap_{i=1}^{N} F\left(T_{i}\right)$, where $q$ is also the unique solution of the variational inequality

$$
\langle(I-f)(q), J(q-p)\rangle \leq 0, \quad \forall p \in \bigcap_{i=1}^{N} F\left(T_{i}\right) .
$$

Proof We divide the proof into the following steps.

Step 1. We show that $\left\{x_{n}\right\}$ is bounded. Let $p \in \bigcap_{i=1}^{N} F\left(T_{i}\right)$. Then $p=B_{n} p$ for all $n \geq 1$ and hence, by the nonexpansiveness of $\left\{B_{n}\right\}_{n=1}^{\infty}$, we have

$$
\begin{aligned}
\left\|x_{n+1}-p\right\| & =\left\|\alpha_{n}\left(f\left(x_{n}\right)-p\right)+\beta_{n}\left(x_{n}-p\right)+\gamma_{n}\left(B_{n} x_{n}-p\right)\right\| \\
& \leq \alpha_{n}\left\|f\left(x_{n}\right)-p\right\|+\beta_{n}\left\|x_{n}-p\right\|+\gamma_{n}\left\|x_{n}-p\right\| \\
& \leq \alpha_{n}\left\|f\left(x_{n}\right)-f(p)\right\|+\alpha_{n}\|f(p)-p\|+\left(1-\alpha_{n}\right)\left\|x_{n}-p\right\| \\
& \leq \alpha_{n} \alpha\left\|x_{n}-p\right\|+\alpha_{n}\|f(p)-p\|+\left(1-\alpha_{n}\right)\left\|x_{n}-p\right\| \\
& =\left(1-\alpha_{n}(1-\alpha)\right)\left\|x_{n}-p\right\|+\alpha_{n}\|f(p)-p\| \\
& \leq \max \left\{\left\|x_{n}-p\right\|, \frac{1}{1-\alpha}\|f(p)-p\|\right\} .
\end{aligned}
$$

By induction, we can conclude that $\left\{x_{n}\right\}$ is bounded. So are $\left\{f\left(x_{n}\right)\right\}$ and $\left\{B_{n} x_{n}\right\}$.

Step 2. We show that $\lim _{n \rightarrow \infty}\left\|x_{n+1}-x_{n}\right\|=0$. To this end, we define $z_{n}=\frac{x_{n+1}-\beta_{n} x_{n}}{1-\beta_{n}}$. From (1.3) we have

$$
\begin{aligned}
\left\|z_{n+1}-z_{n}\right\|= & \left\|\frac{\alpha_{n+1} f\left(x_{n+1}\right)+\gamma_{n+1} B_{n+1} x_{n+1}}{1-\beta_{n+1}}-\frac{\alpha_{n} f\left(x_{n}\right)+\gamma_{n} B_{n} x_{n}}{1-\beta_{n}}\right\| \\
= & \| \frac{\alpha_{n+1}}{1-\beta_{n+1}}\left(f\left(x_{n+1}\right)-B_{n} x_{n}\right)+\frac{\alpha_{n}}{1-\beta_{n}}\left(B_{n} x_{n}-f\left(x_{n}\right)\right) \\
& +\frac{\gamma_{n+1}}{1-\beta_{n+1}}\left(B_{n+1} x_{n+1}-B_{n} x_{n}\right) \| \\
\leq & \frac{\alpha_{n+1}}{1-\beta_{n+1}} M+\frac{\alpha_{n}}{1-\beta_{n}} M+\left\|B_{n+1} x_{n+1}-B_{n} x_{n}\right\| \\
\leq & \left(\frac{\alpha_{n+1}}{1-\beta_{n+1}}+\frac{\alpha_{n}}{1-\beta_{n}}\right) M+\left\|B_{n+1} x_{n+1}-B_{n+1} x_{n}\right\| \\
& +\left\|B_{n+1} x_{n}-B_{n} x_{n}\right\| \\
\leq & \left(\frac{\alpha_{n+1}}{1-\beta_{n+1}}+\frac{\alpha_{n}}{1-\beta_{n}}\right) M+\left\|x_{n+1}-x_{n}\right\|+\left\|B_{n+1} x_{n}-B_{n} x_{n}\right\|
\end{aligned}
$$

for some $M>0$. It turns out that

$$
\left\|z_{n+1}-z_{n}\right\|-\left\|x_{n+1}-x_{n}\right\| \leq\left(\frac{\alpha_{n+1}}{1-\beta_{n+1}}+\frac{\alpha_{n}}{1-\beta_{n}}\right) M+\left\|B_{n+1} x_{n}-B_{n} x_{n}\right\| .
$$


From conditions (C2), (C3) and Lemma 3.4, we have

$$
\limsup _{n \rightarrow \infty}\left(\left\|z_{n+1}-z_{n}\right\|-\left\|x_{n+1}-x_{n}\right\|\right) \leq 0
$$

Lemma 2.3 yields that $\left\|z_{n}-x_{n}\right\| \rightarrow 0$ and hence

$$
\left\|x_{n+1}-x_{n}\right\|=\left(1-\beta_{n}\right)\left\|z_{n}-x_{n}\right\| \rightarrow 0 .
$$

Step 3. We show that $\lim _{n \rightarrow \infty}\left\|B x_{n}-x_{n}\right\|=0$. Indeed, noting that

$$
B_{n} x_{n}-x_{n}=\frac{1}{\gamma_{n}}\left\{\left(x_{n+1}-x_{n}\right)+\alpha_{n}\left(x_{n}-f\left(x_{n}\right)\right)\right\},
$$

we have, by (C2) and (C3),

$$
\lim _{n \rightarrow \infty}\left\|B_{n} x_{n}-x_{n}\right\|=0
$$

Let $B: C \rightarrow C$ be the $B$-mapping generated by $T_{1}, T_{2}, \ldots, T_{N}$ and $\lambda_{1}, \lambda_{2}, \ldots, \lambda_{N}$. So, by Lemma 3.2, we have $B_{n} x \rightarrow B x$ for all $x \in C$. It also follows that

$$
\begin{aligned}
\left\|B x_{n}-x_{n}\right\| & \leq\left\|B x_{n}-B_{n} x_{n}\right\|+\left\|B_{n} x_{n}-x_{n}\right\| \\
& \leq \sup _{z \in\left\{x_{n}\right\}}\left\|B z-B_{n} z\right\|+\left\|B_{n} x_{n}-x_{n}\right\| \\
& \rightarrow 0 .
\end{aligned}
$$

For $t \in(0,1)$, we define a contraction as follows:

$$
S_{t} x=t f(x)+(1-t) B x .
$$

Then there exists a unique path $x_{t} \in C$ such that

$$
x_{t}=t f\left(x_{t}\right)+(1-t) B x_{t} .
$$

From Lemma 2.5, we know that $x_{t} \rightarrow q$ as $t \rightarrow 0$, where $q \in F(B)$. Lemma 3.1(i) also yields that $q \in F(B)=\bigcap_{i=1}^{N} F\left(T_{i}\right)$. Moreover, $q$ is the unique solution of variational inequality (4.1).

Step 4. We show that $\limsup _{n \rightarrow \infty}\left\langle f(q)-q, J\left(x_{n}-q\right)\right\rangle \leq 0$. We see that

$$
x_{t}-x_{n}=(1-t)\left(B x_{t}-x_{n}\right)+t\left(f\left(x_{t}\right)-x_{n}\right) \text {. }
$$

It follows, by Lemma 2.1(ii) that

$$
\begin{aligned}
\left\|x_{t}-x_{n}\right\|^{2} \leq & (1-t)^{2}\left\|B x_{t}-x_{n}\right\|^{2}+2 t\left\langle f\left(x_{t}\right)-x_{n}, J\left(x_{t}-x_{n}\right)\right\rangle \\
\leq & \left(1-2 t+t^{2}\right)\left(\left\|x_{t}-x_{n}\right\|+\left\|B x_{n}-x_{n}\right\|\right)^{2} \\
& +2 t\left\langle f\left(x_{t}\right)-x_{t}, J\left(x_{t}-x_{n}\right)\right\rangle+2 t\left\|x_{t}-x_{n}\right\|^{2},
\end{aligned}
$$


which gives

$$
\left\langle f\left(x_{t}\right)-x_{t}, J\left(x_{n}-x_{t}\right)\right\rangle \leq \frac{\left(1+t^{2}\right)\left\|x_{n}-B x_{n}\right\|}{2 t}\left(2\left\|x_{t}-x_{n}\right\|+\left\|x_{n}-B x_{n}\right\|\right)+\frac{t}{2}\left\|x_{t}-x_{n}\right\|^{2} .
$$

So we have

$$
\limsup _{n \rightarrow \infty}\left\langle f\left(x_{t}\right)-x_{t}, J\left(x_{n}-x_{t}\right)\right\rangle \leq \frac{t}{2} M
$$

for some $M>0$. Since $E$ has a uniformly Gâteaux differentiable norm, $J$ is norm-to-weak* uniformly continuous on bounded subsets of $E$. So we have

$$
\left\langle f(q)-q, J\left(x_{n}-q\right)-J\left(x_{n}-x_{t}\right)\right\rangle \rightarrow 0
$$

and

$$
\left\langle f(q)-f\left(x_{t}\right)+x_{t}-q, J\left(x_{n}-x_{t}\right)\right\rangle \rightarrow 0
$$

as $t \rightarrow 0$. On the other hand, we have

$$
\begin{aligned}
\left\langle f(q)-q, J\left(x_{n}-q\right)\right\rangle= & \left\langle f\left(x_{t}\right)-x_{t}, J\left(x_{n}-x_{t}\right)\right\rangle \\
& +\left\langle f(q)-f\left(x_{t}\right)+x_{t}-q, J\left(x_{n}-x_{t}\right)\right\rangle \\
& +\left\langle f(q)-q, J\left(x_{n}-q\right)-J\left(x_{n}-x_{t}\right)\right\rangle .
\end{aligned}
$$

Since limsup $\operatorname{su}_{n \rightarrow \infty}$ and limsup $\sup _{t \rightarrow 0}$ are interchangeable, using (4.2)-(4.5), we obtain

$$
\limsup _{n \rightarrow \infty}\left\langle f(q)-q, J\left(x_{n}-q\right)\right\rangle \leq 0
$$

Step 5. We show that $x_{n} \rightarrow q$ as $n \rightarrow \infty$. In fact, we have

$$
\begin{aligned}
\left\|x_{n+1}-q\right\|^{2}= & \alpha_{n}\left\langle f\left(x_{n}\right)-q, J\left(x_{n+1}-q\right)\right\rangle+\beta_{n}\left\langle x_{n}-q, J\left(x_{n+1}-q\right)\right\rangle \\
& +\gamma_{n}\left\langle B_{n} x_{n}-q, J\left(x_{n+1}-q\right)\right\rangle \\
\leq & \alpha_{n} \alpha\left\|x_{n}-q\right\|\left\|x_{n+1}-q\right\|+\alpha_{n}\left\langle f(q)-q, J\left(x_{n+1}-q\right)\right\rangle \\
& +\beta_{n}\left\|x_{n}-q\right\|\left\|x_{n+1}-q\right\|+\gamma_{n}\left\|x_{n}-q\right\|\left\|x_{n+1}-q\right\| \\
= & \left(1-\alpha_{n}(1-\alpha)\right)\left\|x_{n}-q\right\|\left\|x_{n+1}-q\right\|+\alpha_{n}\left\langle f(q)-q, J\left(x_{n+1}-q\right)\right\rangle \\
\leq & \frac{1}{2}\left(1-\alpha_{n}(1-\alpha)\right)\left(\left\|x_{n}-q\right\|^{2}+\left\|x_{n+1}-q\right\|^{2}\right)+\alpha_{n}\left\langle f(q)-q, J\left(x_{n+1}-q\right)\right\rangle,
\end{aligned}
$$

which implies that

$$
\begin{aligned}
\left\|x_{n+1}-q\right\|^{2} \leq & \frac{1-\alpha_{n}(1-\alpha)}{1+\alpha_{n}(1-\alpha)}\left\|x_{n}-q\right\|^{2}+\frac{2 \alpha_{n}}{1+\alpha_{n}(1-\alpha)}\left\langle f(q)-q, J\left(x_{n+1}-q\right)\right\rangle \\
= & \left(1-\frac{2 \alpha_{n}(1-\alpha)}{1+\alpha_{n}(1-\alpha)}\right)\left\|x_{n}-q\right\|^{2} \\
& +\frac{2 \alpha_{n}}{1+\alpha_{n}(1-\alpha)}\left\langle f(q)-q, J\left(x_{n+1}-q\right)\right\rangle .
\end{aligned}
$$


Put $c_{n}=\frac{2 \alpha_{n}(1-\alpha)}{1+\alpha_{n}(1-\alpha)}$ and $b_{n}=\frac{2 \alpha_{n}}{1+\alpha_{n}(1-\alpha)}\left\langle f(q)-q, J\left(x_{n+1}-q\right)\right\rangle$. So it is easy to check that $\left\{c_{n}\right\}$ is a sequence in $(0,1)$ such that $\sum_{n=1}^{\infty} c_{n}=\infty$ and $\limsup _{n \rightarrow \infty} \frac{b_{n}}{c_{n}} \leq 0$. Hence, by Lemma 2.7, we conclude that $x_{n} \rightarrow q$ as $n \rightarrow \infty$. This completes the proof.

\section{Competing interests}

The author declares that he has no competing interests.

\section{Acknowledgements}

The author wishes to thank editor/referees for valuable suggestions and Professor Suthep Suantai for the guidance. This research was supported by the Thailand Research Fund, the Commission on Higher Education, and University of Phayao under Grant MRG5580016.

Received: 16 April 2013 Accepted: 5 July 2013 Published: 22 July 2013

\section{References}

1. Mann, WR: Mean value methods in iteration. Proc. Am. Math. Soc. 4, 506-510 (1953)

2. Reich, S: Weak convergence theorems for nonexpansive mappings in Banach spaces. J. Math. Anal. Appl. 67, 274-276 (1979)

3. Ishikawa, S: Fixed points by a new iteration method. Proc. Am. Math. Soc. 44, 147-150 (1974)

4. Agarwal, RP, O'Regan, D, Sahu, DR: Iterative construction of fixed points of nearly asymptotically nonexpansive mappings. J. Nonlinear Convex Anal. 8,61-79 (2007)

5. Agarwal, RP, O'Regan, D, Sahu, DR: Fixed Point Theory for Lipschitzian-Type Mappings with Applications. Springer, New York (2009)

6. Atsushiba, S, Takahashi, W: Strong convergence theorems for a finite family of nonexpansive mappings and applications. Indian J. Math. 41, 435-453 (1999). In: B.N. Prasad birth centenary commemoration volume

7. Bruck, RE: Properties of fixed-point sets of nonexpansive mappings in Banach spaces. Trans. Am. Math. Soc. 179, 251-262 (1973)

8. Kangtunyakarn, A, Suantai, S: A new mapping for finding common solutions of equilibrium problems and fixed point problems of finite family of nonexpansive mappings. Nonlinear Anal. TMA 71, 4448-4460 (2009)

9. Takahashi, W, Shimoji, K: Convergence theorems for nonexpansive mappings and feasibility problems. Math. Comput. Model. 32, 1463-1471 (2000)

10. Moudafi, A: Viscosity approximation methods for fixed point problems. J. Math. Anal. Appl. 241, 46-55 (2000)

11. Chang, SS: Viscosity approximation methods for a finite family of nonexpansive mappings in Banach spaces. J. Math. Anal. Appl. 323, 1402-1416 (2006)

12. Cho, YJ, Kang, SM, Zhou, H: Some control conditions on iterative methods. Commun. Appl. Nonlinear Anal. 12, 27-34 (2005)

13. $\mathrm{Hu}, \mathrm{L}-\mathrm{G}$ : Theorems of strong convergence of mixed iterative methods for obtaining strict pseudocontractions in Banach spaces. Appl. Math. Lett. 23, 791-795 (2010)

14. Jung, JS: Iterative approaches to common fixed points of nonexpansive mappings in Banach spaces. J. Math. Anal. Appl. 302, 509-520 (2005)

15. Jung, JS, Sahu, DR: Convergence of approximating paths to solutions of variational inequalities involving non-Lipschitzian mappings. J. Korean Math. Soc. 45, 377-392 (2008)

16. Marino, G, Xu, HK: A general iterative method for nonexpansive mappings in Hilbert spaces. J. Math. Anal. Appl. 318 43-52 (2006)

17. Takahashi, W: Viscosity approximation methods for countable families of nonexpansive mappings in Banach spaces. Nonlinear Anal. TMA 70, 719-734 (2009)

18. Xu, HK: Viscosity approximation methods for nonexpansive mappings. J. Math. Anal. Appl. 298, $279-291$ (2004)

19. Xu, HK: Another control condition in iterative method for nonexpansive mappings. Bull. Aust. Math. Soc. 65, 109-113 (2002)

20. Yao, Y, Liou, LC, Zhou, H: Strong convergence of an iterative method for nonexpansive mappings with new control conditions. Nonlinear Anal. TMA 70, 2332-2336 (2009)

21. Zhou, H, Wei, L, Cho, YJ: Strong convergence theorems on an iterative method for a family of finite nonexpansive mappings in reflexive Banach spaces. Appl. Math. Comput. 173, 196-212 (2006)

22. Opial, Z: Weak convergence of successive approximations for nonexpansive mappings. Bull. Am. Math. Soc. 73 591-597 (1967)

23. Browder, FE: Nonexpansive nonlinear operators in a Banach space. Proc. Natl. Acad. Sci. USA 54, 1041-1044 (1965)

24. Takahashi, W: Nonlinear Function Analysis. Yokohama Publishers, Yokohama (2000)

25. Suzuki, T: Strong convergence of Krasnoselskii and Mann's type sequences for one parameter nonexpansive semigroups without Bochner integrals. J. Math. Anal. Appl. 305, 227-239 (2005)

26. Tan, KK, Xu, HK: Fixed point iteration processes for asymptotically nonexpansive mappings. Proc. Am. Math. Soc. 122 733-739 (1994)

27. Bruck, RE: A simple proof of the mean ergodic theorem for nonlinear contractions in Banach spaces. Isr. J. Math. 32 107-116 (1979) 